\title{
Application of Electronic Structure Methods to Coupled Drude Oscillators
}

\author{
T. T. Odbadrakh ${ }^{\mathrm{a}}$, V. Voora ${ }^{\mathrm{a}, 1}$, and K. D. Jordan ${ }^{*, a}$ \\ ${ }^{a}$ Department of Chemistry, University of Pittsburgh, Pittsburgh, PA 15260, USA
}

\begin{abstract}
The configuration interaction, perturbation theory, coupled cluster doubles, and random phase approximation methods are applied to a system of two quantum Drude oscillators interacting through dipole-dipole coupling. It is found that the random phase approximation gives the exact excitation energies and exact interaction energy of this system even when allowing only excitations into the first excited levels of the oscillators. In contrast, to obtain the exact results from configuration interaction or coupled cluster treatments of the model requires inclusion of excitations into all accessible excited configurations.
\end{abstract}

${ }^{1}$ Present address: Department of Chemistry, University of California, Irvine, CA 92697, USA.

*Corresponding author. Email: jordan@ pitt.edu, tel: 412-624-8690. 


\section{Introduction}

A Drude oscillator consists of two fictitious charges $+q$ and $-q$, coupled harmonically through a force constant $k{ }^{1}$ One charge is held fixed (here assumed to be the positive charge), and the other is displaceable. Treated classically, Drude oscillators can describe polarization interactions, and, when treated quantum mechanically, they can also be used to describe dispersion interactions between atoms or molecules. In recent years Drude oscillators have been employed in several force fields, ${ }^{2-5}$ generally to describe polarization, and in approaches for correcting DFT for long-range dispersion interactions. ${ }^{6}$ They have also been used in our group in a QM/MM approach for modeling an excess electron interacting with water clusters. ${ }^{7,8}$

The system of two Drude oscillators, separated by a distance $R$, interacting via a dipole-dipole coupling is a textbook model for explaining the origin of $C_{6} R^{-6}$ dispersion interaction between two atoms or molecules. ${ }^{9}$ The dispersion interaction between the two oscillators can be solved analytically by a change of variables. Here, we find it instructive to apply standard electronic structure methods including Rayleigh-Schrodinger perturbation theory, ${ }^{10}$ configuration interaction (CI), coupled cluster doubles (CCD) theory, ${ }^{11,12}$ and the random phase approximation (RPA) ${ }^{13-15}$ to calculate the interaction energy between two Drude oscillators. The configurations used in the calculations are represented in terms of products of harmonic oscillator functions. Our analysis shows that while the CI and CCD methods require including excitations into all excited levels to obtain the exact interaction energy, the RPA method gives the exact answer, allowing only excitations into the first excited state of each oscillator. The insensitivity of the RPA result to the basis set is explained in terms of the Hamiltonian being quadratic in nature.

\section{Methodology:}

The Hamiltonian for the model system is given by

$$
H=\frac{1}{2} \frac{d^{2}}{d x_{1}^{2}} \quad \frac{1}{2} \frac{d^{2}}{d x_{2}^{2}}+\frac{1}{2} k x_{1}^{2}+\frac{1}{2} k x_{2}^{2} \quad \frac{2 q^{2} x_{1} x_{2}}{R^{3}}
$$


where, for simplicity, we assume one-dimensional oscillators and employ atomic units. We have further assumed the mass associated with the oscillator is equal to that of the electron, which is one in atomic units. We recast $H$ as:

$$
H=H_{0}+V
$$

where $H_{0}$ is the Hamiltonian for the non-interacting oscillators and the perturbation $V$ is defined as

$$
V=\frac{2 q^{2} x_{1} x_{2}}{R^{3}}=x_{1} x_{2}
$$

With the change of variables

$$
=\frac{1}{\sqrt{2}}\left(x_{1}+x_{2}\right)
$$

and

$$
=\frac{1}{\sqrt{2}}\left(\begin{array}{ll}
x_{1} & x_{2}
\end{array}\right),
$$

$H$ separates to give two non-interacting harmonic oscillators with frequencies

$$
{ }_{1}=\sqrt{1 \frac{-}{k}}
$$

and

$$
{ }_{2}=\sqrt{1+\frac{-}{k}}
$$

where is the frequency of an unperturbed oscillator. The change in zero-point energy of the system due to the interaction between the oscillators is

$$
E_{\mathrm{int}}=\frac{-}{2}\left[2+\sqrt{1+\frac{-}{k}}+\sqrt{1 \frac{\bar{k}}{k}}\right] .
$$

Taylor series expansion of the square roots gives

$$
E_{\text {int }}=\frac{2}{8 k^{2}} \frac{5 \quad 4}{128 k^{4}}
$$


through the first two non-zero terms. This can be rewritten as

$$
E_{\mathrm{int}}=\frac{1}{2}{ }^{2} \frac{R^{6}}{8}{ }^{4} \frac{}{R^{12}}
$$

where use was made of the fact that the polarizability, , of a Drude oscillator is $q^{2} / k$. The first term is the well-known London expression for the leading dispersion interaction between two atoms or molecules. $^{16}$

III. Application of electronic structure methods to the Drude oscillator problem

We now apply various electronic structure methods to the Hamiltonian given by Eq. 1. The basis set is taken to be products of harmonic oscillator functions $|i j\rangle$, where $i$ and $j$ specify the quantum numbers of the two oscillators.

A. Configuration Interaction, Rayleigh-Schrodinger perturbation theory, and CCD

The CI matrix for the ground state of the coupled oscillator problem assumes the form

$$
\begin{aligned}
& |00\rangle|11\rangle \quad|20\rangle \quad|02\rangle \quad|22\rangle \quad \ldots
\end{aligned}
$$

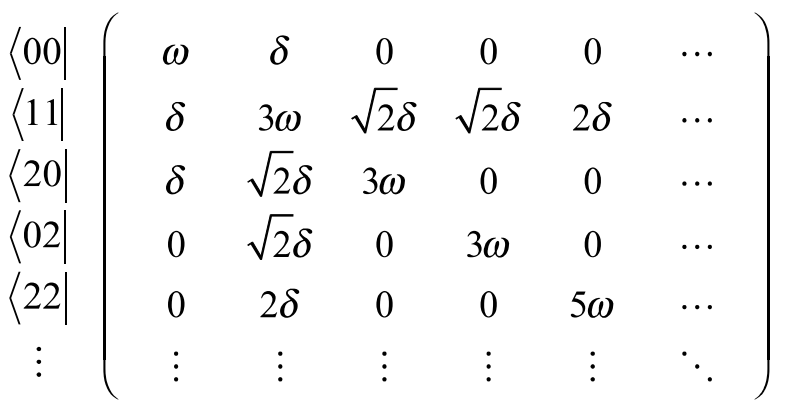

where $=/(2)$. As seen from the CI matrix the configurations that contribute to the ground state wave function are $|00\rangle,|11\rangle,|20\rangle,|02\rangle,|22\rangle$, etc. Note that the $|00\rangle$ configuration mixes directly only with $|11\rangle$. If only the $|00\rangle$ and $|11\rangle$ configurations are retained, the energy is

$$
2 \sqrt{1+\frac{2}{2}}=\frac{2}{2}+\frac{4}{83^{3}} \square=\frac{2}{8 k^{2}}+\frac{4}{128 k^{4}}
$$


which is correct only through the leading correction, i.e., the second-order perturbation theory result. In fact, the $O\left({ }^{4}\right)$ term enters with the wrong sign. As is clear from the structure of the CI matrix, the $|20\rangle$, $|02\rangle$, and $|22\rangle$ configurations must also be included to obtain the energy correct through fourth-order perturbation theory. There are four contributions to the fourth-order energy:

$$
\begin{aligned}
& \frac{\langle 00|V| 11\rangle\langle 11|V| 22\rangle\langle 22|V| 11\rangle\langle 11|V| 00\rangle}{\left(E_{00} \quad E_{11}\right)^{2}\left(E_{00} \quad E_{22}\right)} \\
& \frac{\langle 00|V| 11\rangle\langle 11|V| 20\rangle\langle 20|V| 11\rangle\langle 11|V| 00\rangle}{\left(E_{00} \quad E_{11}\right)^{2}\left(E_{00} \quad E_{20}\right)} \\
& \frac{\langle 00|V| 11\rangle\langle 11|V| 02\rangle\langle 02|V| 11\rangle\langle 11|V| 00\rangle}{\left(E_{00} \quad E_{11}\right)^{2}\left(E_{00} \quad E_{02}\right)} \\
& \frac{\langle 00|V| 11\rangle\langle 11|V| 00\rangle\langle 00|V| 11\rangle\langle 11|V| 00\rangle}{\left(E_{00} \quad E_{11}\right)^{3}}
\end{aligned}
$$

The terms involving $|20\rangle,|02\rangle$, and $|22\rangle$ each contribute ${ }^{4} / 4^{3}$ while the disconnected diagram derived from $|\langle 00|V| 11\rangle|^{4} / 8{ }^{3}$ contributes ${ }^{4} / 8{ }^{3}$.

Fig. 1 reports the interaction energy vs. distance for the coupled Drude oscillator problem in the case $\mathrm{k}=0.04$ a.u., $\mathrm{q}=1$ a.u., and $\mathrm{m}=1$ a.u. (This corresponds to an oscillator excitation energy of about $5.4 \mathrm{eV}$.) Results are reported for three different CI calculations: the smallest including only the $|00\rangle$ and $|11\rangle$ configurations, the intermediate including the $|00\rangle,|11\rangle,|20\rangle,|02\rangle$, and $|22\rangle$ configurations, and the largest including the $|00\rangle,|11\rangle,|20\rangle,|02\rangle,|22\rangle,|13\rangle,|31\rangle$, and $|33\rangle$ configurations. Over the range of $R$ values considered ( $4 \leq R \leq 6$ a.u.) the largest CI calculation gives interaction energies essentially indistinguishable from the exact values, while the results from the intermediate size CI calculations differ slightly from the exact result for $R \leq 4.5$ a.u. On the other hand, the energies from the small CI calculations differ appreciable from the exact result for $R$ values less than about 5 a.u. For the 
two-Drude oscillator model system, the CCD method ${ }^{10,11}$ gives the same result as the CI calculations, when using the same configuration space. For this reason, the CCD method is not pursued further.

\section{B. RPA}

As is well known the RPA equations can be written as

$$
\left(\begin{array}{cc}
\underline{\underline{A}} & \underline{\underline{B}} \\
\underline{\underline{B}} & \underline{\underline{A}}
\end{array}\right)\left(\begin{array}{c}
\underline{x} \\
\underline{y}
\end{array}\right)=E\left(\begin{array}{ll}
1 & 0 \\
0 & 1
\end{array}\right)\left(\begin{array}{l}
\underline{x} \\
\underline{y}
\end{array}\right)
$$

where the $A$ matrix includes interactions between various single excitations and the $B$ matrix accounts for the mixing of double excitations with the reference configuration. ${ }^{13-15}$ Due to the nature of the RPA equations, the only configurations that enter the $A$ matrix for the model considered here are $|10\rangle$ and $|01\rangle$.

The entries in the $A$ matrix are $\left\langle 10\left|H \quad E_{0}\right| 10\right\rangle=\left\langle 01\left|H \quad E_{0}\right| 01\right\rangle=\quad$ and $\left\langle 10\left|H \quad E_{0}\right| 01\right\rangle=$, while the non-zero entries in the $B$ matrix are $\langle 11|H| 00\rangle=\langle 00|H| 11\rangle=$.

Thus, the $A$ and $B$ matrices are

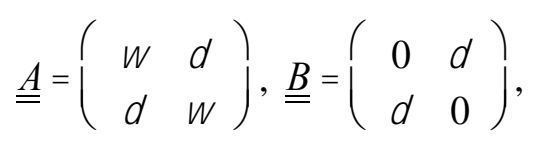

and the relevant excitation energies are

$$
E_{1}=\sqrt{1+\frac{2}{2}}
$$

and

$$
E_{2}=\sqrt{1 \frac{2}{2}}
$$

Several different strategies have been developed for extracting ground state correlation energies of systems where the electrons are treated explicitly. ${ }^{14,17-20}$ For the Drude oscillator problem, the logical choice is to use

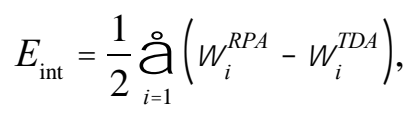


where TDA refers to the Tamm-Dancoff approximation which retains only the A matrix in Eq. 12. For the coupled Drude oscillator problem with only $|0\rangle \rightarrow|1\rangle$ excitations of the individual oscillators, this gives

$$
E_{\mathrm{int}}=\frac{-}{2}\left[2+\sqrt{1+\frac{2}{2}}+\sqrt{1 \frac{2}{2}}\right]
$$

which is identical to the exact solution given by Eq. 8. At first sight, this is a surprising result, since obtaining the exact correlation energy with the CI or CCD methods requires inclusion of all configurations $|i j\rangle$, where $|i+j|$ is an even integer. It is also interesting in light of the fact that it has been shown that the direct RPA method is equivalent to the ring CCD method (without exchange) for estimating the correlation energy of a many-electron system. ${ }^{21-23}$

Comparison of Eqs. 14 and 15 with Eqs. 6 and 7 reveals that the RPA method allowing only $|0\rangle \rightarrow|1\rangle$ excitations of the oscillators gives the exact excitation energies of the coupled Drude oscillator problem. It is for this reason that Eq. 16 gives the exact value for the ground state correlation energy when using the restricted excitation space. This raises the question as to the structure of the A and $\mathrm{B}$ matrices when excitations into levels $|2\rangle,|3\rangle$, etc., are allowed. In this case, the eigenvalues of the A matrix become $n$ where $n$ is the quantum number of the excitation. Moreover, the fact that the RPA excitation energies are exact when allowing only $|0\rangle \rightarrow|1\rangle$ excitations of the oscillators follows from the quadratic form of the Hamiltonian as can be seen from the derivation of the RPA equations by Rowe. ${ }^{24}$ Additionally, we note that the fact that the RPA method gives the exact correlation energy for harmonic oscillators coupled through the dipole-dipole interaction, has been demonstrated by Tkatchenko and coworkers $^{25}$ by use of the adiabatic connection fluctuation-dissipation theorem (AC-FDT). ${ }^{26}$ Interestingly, even though Tkatchenko et al. did not explicitly introduce a basis set in their treatment, they did employ the functional form of $\quad(\quad)$, and the polarizability of a Drude oscillator depends only on the $|0\rangle \rightarrow|1\rangle$ transition. If the Hamiltonian were extended to include also dipole-quadrupole and quadrupole- 
quadrupole coupling, $|0\rangle \rightarrow|2\rangle$ excitations would also enter into the RPA equations, and the method would no longer give the exact excitation energies nor the exact ground state energy.

Examination of the Goldstone diagrams at each order of the RPA correlation energy reveals that at all orders other than second all diagrams contributing to the energy are exclusion principle violating (EPV) in nature. This is illustrated in Fig. 2, which displays one of the fourth-order contributions to the energy. This is in contrast to the RSPT result for which the negative contributions to the correlation energy are non-EPV in nature and include excitations into $|20\rangle,|02\rangle$, and $|22\rangle$.

\section{CONCLUSIONS:}

Quantum Drude oscillators have proven to be a valuable approach for describing dispersion interactions in molecules, clusters, and solids as well as at interfaces. In this work the interaction energies for a pair of interacting quantum Drude oscillators with dipole-dipole coupling is calculated using the perturbation theory, CI, CCD, and RPA methods. The RPA method is shown to give the exact excitation energies and the exact correlation energy of the ground state of the coupled Drude oscillator problem when allowing only $|0\rangle \rightarrow|1\rangle$ excitations of the oscillators. This lack of sensitivity to the basis set (and, hence, excitation level) is a consequence of the Hamiltonian including only dipole-dipole coupling of the oscillators. It also follows from the result of Tkatchenko and co-workers who showed using the AC-FDT that the RPA gives the exact interaction energy for a system of oscillators interacting through dipoledipole coupling. 


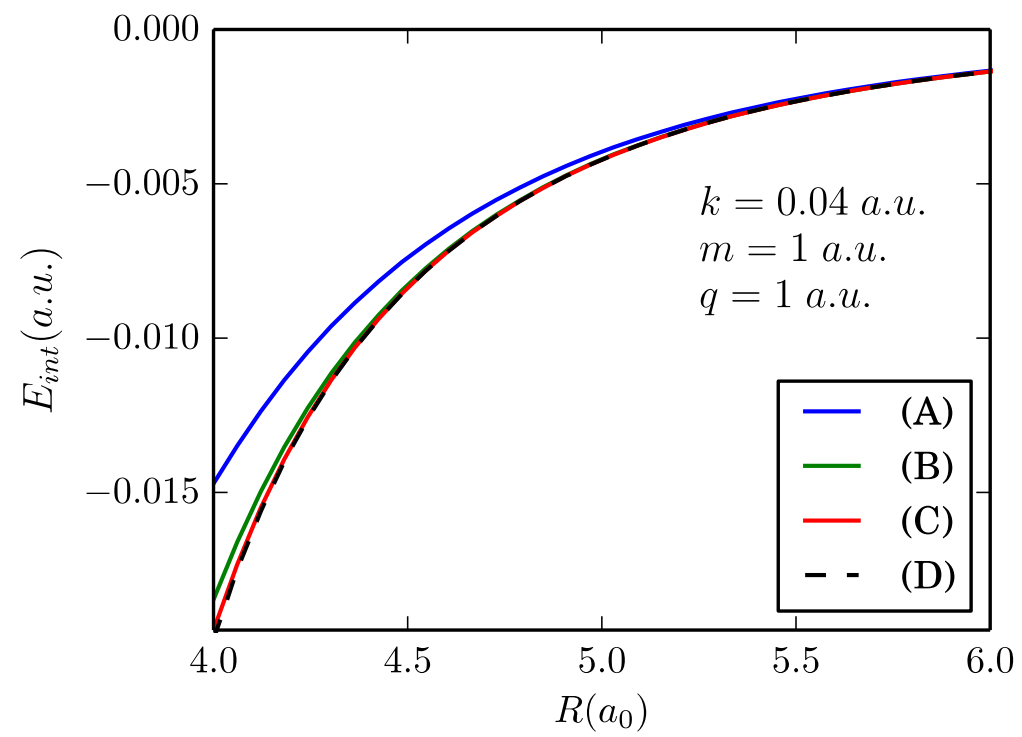

Figure 1. Interaction energy of two Drude oscillators with dipole-dipole coupling calculated with configuration interaction with basis (A) $|00\rangle,|11\rangle$; (B) $|00\rangle,|11\rangle,|20\rangle,|02\rangle,|22\rangle$; and (C) $|00\rangle,|11\rangle$, $|20\rangle,|02\rangle,|22\rangle,|13\rangle,|31\rangle,|33\rangle$, and compared to (D) the exact interaction energy. 


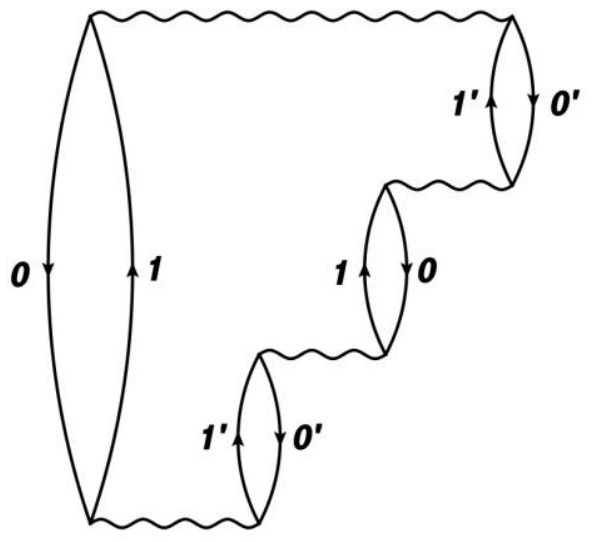

(A)

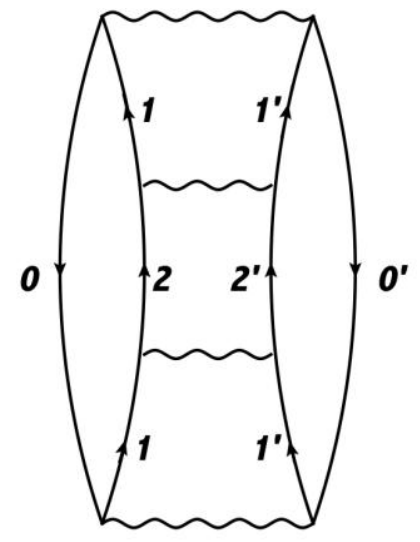

(B)

Figure 2. (A) Fourth-order exclusion principle violating interaction diagram appearing in the RPA. $|0\rangle$ and $|1\rangle$ correspond to oscillator 1, while $\left|0^{\prime}\right\rangle$ and $\left|1^{\prime}\right\rangle$ correspond to oscillator 2. (B) Shows a fourth-order contribution to the RSPT involving excitation into the $|22\rangle$ level. 
ACKNOWLEGEMENTS: This research was carried out with the support of a grant CHE1362334 from the National Science Foundation. We acknowledge valuable discussions with Filipp Furche and Andreas Hesselmann.

\section{References}

1. P. Drude, Lehrbuch der Optik (S. Hirzel, Leipzig, 1900).

2. G. Lamoureux, J. Chem. Phys. 119, 5188 (2003).

3. A. Jones, A. Thompson, J. Crain, M. Muser, and G. Martyna, Phys. Rev. B 79, 1 (2009).

4. W. Jiang, D. J. Hardy, J. C. Phillips, A. D. Mackerell Jr., K. Schulten, and B. Roux, J. Phys. Chem. Lett. 2, 87 (2011).

5. H. Yu, and W. F. van Gunsteren, J. Chem. Phys. 121, 9549 (2004).

6. A. Tkatchenko, R. A. DiStasio, R. Car, and M. Scheffler, Phys. Rev. Lett. 108, 236402 (2012).

7. F. Wang and K. D. Jordan, J. Chem. Phys. 114, 10717 (2001).

8. F. Wang and K. D. Jordan, J. Chem. Phys. 116, 6973 (2002).

9. A. R. Leach, Molecular Modeling, Addison Wesley.

10.E. Schrodinger, Annalen de Physik, 80, 437 (1926); J. W. S. Rayleigh, Theory of Sound, pp 115-118 (London, McMillan, 1894).

11. F. Coester and H. Kummel, Nucl. Phys. 17, 477 (1960).

12. J. Cizek, J. Chem. Phys. 45, 4256 (1966)

13. D. J. Thouless, Nucl. Phys. 22, 78 (1961).

14. A. D. McLachlan and M. A. Ball, Rev. Mod. Phys. 36, 844 (1964).

15. H. Eshuis, J. E. Bates, and F. Furche, Theor. Chem. Acc. 1311084 (2012).

16. F. London, Z. Physik 63, 245 (1930).

17. A. Szaebo and N. S. Ostlund, J. Chem. Phys. 67, 4351 (1977).

18. J. Oddeshedde, Adv. Quant. Chem. 11, 275 (1978).

19. N. Fukada, F. Iwamoto, and K. Sawada, Phys. Rev. 135, A932 (1964).

20. A. Hesselmann, J. Chem. Phys.134, 204107 (2011). 
21. J. Toulouse, W. Zhu, A. Savin, G. Jansen, and J. G. Angyan, J. Chem. Phys. 135, 084119 (2011).

22. G. Scuseria, T. M. Henderson, and D. C. Sorensen, J. Chem. Phys. 129, 231101 (2008).

23. R. Moszynski, B. Jeziorski, and K. Szalewicz, Int. J. Quant. Chem. 45, 409 (1993)

24. D. J. Rowe, Rev. Mod. Phys. 40, 153 (1968).

25 R. A. Tkatchenko, A. Ambrosetti, and R. A. DiStasio, Jr., J. Chem. Phys. 138, 074106 (2013).

26. D. C. Langreth and J. C. Perdew, Phys. Rev. B 15, 2884 (1977). 

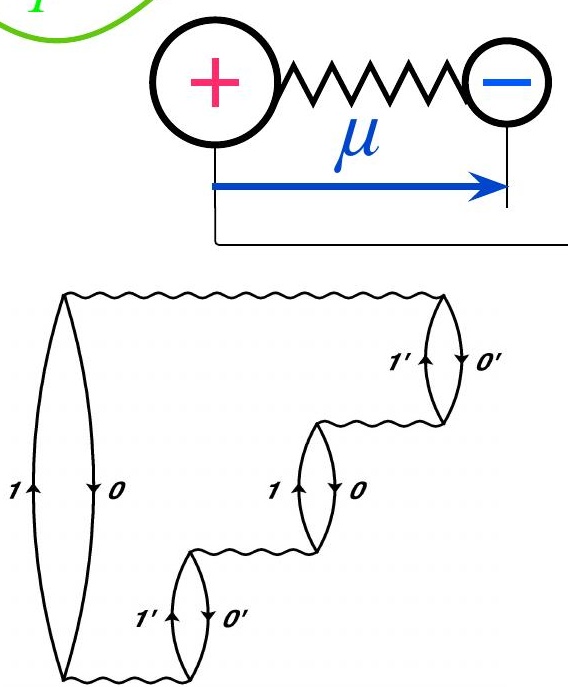

$$
\begin{gathered}
R \\
E_{\text {int }}=-\frac{1}{2} \alpha^{2} \frac{\omega}{R^{6}}-\frac{5}{8} \alpha^{4} \frac{\omega}{R^{12}}-\ldots
\end{gathered}
$$$$
\underset{\mu^{\prime}}{\longrightarrow}
$$

Graphical Abstract 